\title{
Strengthening public health research for improved health
}

\section{J Selva Andina Res Soc. 2012; 3(1):1-2.}

Research in public health is a range that includes from fundamental research to research in clinical practice, including novel advances, evaluation of results and their spreading. Actually, public health research is considered multidisciplinary incorporating numerous factors in its development. Establishing as a mainstay the scientific method, deepens in basic research, clinical epidemiological research and health services. The premise of quality and relevance is reflected in international scientific research, and in the daily work and good biomedical practices that should be included in the research as a common task. Therefore, the research must take a proactive stance of inquiry, integrating a concern planned and ongoing development of knowledge. This requires improve international coordination, seeking a balance between basic and applied research as well as science and technology. Thus research cannot be considered without innovation, weighing up the people and society needs.

Acting on knowledge of scientific production processes requires greater procedures thoroughness and the effective expression of the results. It is noted as essential to establish explicit principles in review and evaluation of the adjustments of actions, always within the standards of scientific conduct and fairness of the research process. In the biomedical scientific lines it have to be consider general assessments that occur related to the impact and quality of health research, mostly leading efforts to areas that require further attention. However, other subject areas that may be deficient or with lower incidence in the population should not be overlook.

Health research as a source of new applications and development provides knowledge, improving well-being. However, it is understandable without considering the needs and social demands. Therefore, in public health research and to improve the health of the population, we must refine and optimize the prevention and prophylaxis methods. The multidisciplinary and multi-center approach in research will provide a better understanding of the processes and quality solutions. The implementation of strategies that encourage the promotion of research will lead to the establishment of joint action lines, allowing a general approach in enhancing biomedical research. In this sense and for social improvement, awareness of researchers in encouraging the detection of social problems is especially relevant.

As mentioned it's estimate the need for establish an adequate framework for public health research in loss-making countries, with results that impact on the advancement of the welfare of the people, advocating to take appropriate actions by the governments and health authorities. Therefore, the primary purpose must be to protect and improve the health of people. This specific aim is positioned on the border between basic research and development, so the contribution of ideas from clinical practice should be used in the treatment of health problems and advance of the 
prevention. At the same time, promotion of public health training habits will contribute to a better knowledge transfer and implementation of healthy behaviors to collaborate towards the development. There's an extraordinary opportunity for the establishment of public health research, through the primary consideration of major health problems and providing workable solutions that contribute to improve the existing situation. Overcoming health challenges undoubtedly lead to advance in sustainability in the twenty-first century, producing a social benefit, promoting the progress of humanity in technological and communicative processes, and equity. The competition between research groups should be understood as a mechanism for constructive approach with the ultimate aim to improve society. In turn, the latter must understand and appreciate the progress made through biomedical research, so an effort to scientific communication and dissemination should be made, ensuring the reliability of scientific research. In particular, it will serve a better understanding of research results, mainly those whose objective assessment of social benefits is delayed or complex. In the process of public health research the most efficient way must be achieved (well understood) with rigor and ethics, in order to prevent diseases, protect and promote health, and technology upgrading among others; pursuing like the ultimate goal to attain a better quality of life of the population.

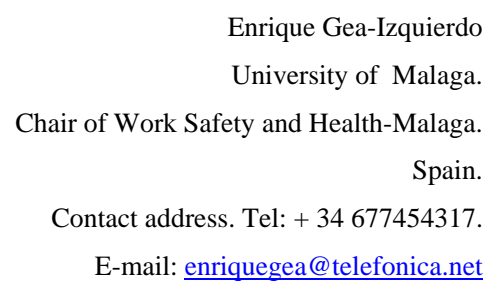

(C) 2012. Journal of the Selva Andina Research Society. Bolivian. All rights reserved 